\title{
EXPLORING THE ETHICAL DILEMMAS OF AFRO-CENTRIC SOCIAL MEDIA USE THROUGH AGENT-BASED MODELING: A REVIEW
}

\author{
C. H. NwoKoye*, I. I. Umeh AND I. J. Odo \\ (C. H. N.: Open Studies Unit, Nigerian Correctional Service, Awka, \\ Anambra State, Nigeria; I. I. U: Computer Science Department, Nnamdi Azikiwe \\ University, Nigeria. I. J. O.: Computer Science Department, Chukwuemeka Odumegwu \\ Ojukwu University, Nigeria). \\ Corresponding author's email: chinonsonwokoye@gmail.com
}

\begin{abstract}
Social media (SM) has become indispensable for individuals and workplaces/organizations in Africa and beyond. Therein, ethical concerns are posed due to the inability to create virtual boundaries (VM), the intractability of guidelines for managers and other unintended risks/consequences. Operations research was used for modeling ethical concerns but have been defeated due to reasons of several ethical values and various assessment criteria for stakeholders. Consequently, this review paper initially x-rays the import of ethical dilemmas in older studies so as to conceive a strategy characterized by engaging stakeholders that utilize SM via Agent-Based Modeling (ABM), in such a manner that ethics can be evaluated. Additionally, it presented the rudiments of social media ABM explorations and the peculiarities of Africa. Finally, the review provided a suitable methodology and sheds light on the possible challenges of ABM implementation. Besides the benefit of increased patronage, the agent technology may also constitute a pedagogical tool for learning ethical behavior. Moreover, it is our hope that with the involvement of experts of related disciplines in Africa, attendant theories are formalized and used for building agent models that allows ethical decision making, weighing of pros and cons, analyzing differences and dimensions inherent in VM creation.
\end{abstract}

Keywords: Social media, ethics, Agent Based Modeling, Africa

\section{Introduction}

Ethical values are at the core of the continued existence of mankind in modern societies plagued by myriads of problems. The implication is that having ethical issues in mind, advancements technological and otherwise, may not inhibit the addressing of future needs in trying to address current problems. In a nut shell, ethics ensures sustainable development (Kunsch et al., 2007; Kunsch, et al., 2009). As it relates to social media, Voshel \& Wesala (2015) maintain that, "the inherent ethical implications need to be a guiding factor related to the creation of a social media identity and thus serve as a person's "social conscience".

Social networking sites (SNS) overlap networks of partners in several work circles, friends and family in unanticipated ways, contrary to networks in real life where individuals and their interactions may be separated by distance, time, religion, belief system etc. This defies moves to create virtual boundaries (Kimball \& Kim, 2013). Social media use involves interactions using the internet for the creation and distribution of various contents. Therefore, as the world 
witness a continually overwhelming emergence of several social computing technologies there has also been a parallel modification in communication conventions and processes of thought. This is verifiably true for Africa if one considers the ubiquitous social networking environments such as Twitter, Facebook etc. These platforms currently intrigue and actively engage young adults or the Generation Y people born after 1981 (Bolton et al., 2013) as well as subsequent generations.

Additionally,"...the gratifications of using these sites tend to outweigh the perceived threats of privacy" (Debatin, et al., 2009) or any other concern. For the benefit of customers or consumers, companies employ networking platforms in order to create a "promotional mix of advertising, personal selling, public relations, publicity, direct marketing and sales promotion" (Mangold \& Faulds, 2009). In this case, social mediabased conversations or interactions can get of control of the manager. Some industry experts have recommended the ban of social media but this may result to hindrances for the company in the future. For instance, most recently the Federal Government of Nigeria banned the use of Twitter (Ewokor, 2021) for the masses and this prompted even more online assaults and altercations in the country. These circumstances beg for policies that will ethically guide social media interactions in network arenas (Kimball \& Kim, 2013), to meet organizational performance objectives. As part of their professional practice, managers and other stake holders (IT specialists, legal officers and human resource) must be prepared to address social media and its attendant issues. Therefore, their job should be simplified by applying agent technology. This would aid their decision making process. Interestingly, the rationale for proposing agent based modeling (ABM) can be traced to Bonabeau (2002), who extended it to an organizational setting, thus confirming several events that require this technology. Specifically, ABM is required when natural system descriptions depends on the outlook and activities constituting elements, when the combined transition rates of mathematical equations on the subject does not distinctly describe individual behavior, when individual behavior is both complex and heterogeneous, when activities are a more natural way of describing the system than processes and when stochasticity applies to stakeholder behaviors in the system.

\section{Ethical issues, ABM and social media use}

Operations Research (OR) methods have been used to model ethical concerns for policy makers who must be abreast of all dimensions of their work life. However, it was found that the introduction of ethical issues in management and social sciences tends to defeat OR techniques used for optimization problems (Azar \& Sadeghi, 2012). Another reason for this defeat is that modeling ethical problems may involve various stakeholders coming from various disciplines with various ethical values and various assessment criteria. This gave rise to the promotion of systemic disciplines such as ABM, which aims at "gaining insights into complex systemic structures" (Kunsch, et al., 2009). In relation to our discourse, ABM - a sub-discipline of Artificial Intelligence and Distributed Artificial Intelligence achieves "insight into ethical values and behaviors" (Danielson, 2003). This is because the approach provides a rapid and adaptable method for characterizing agents, their abode and their inherent interrelations. This modeling approach provides opportunities for naturally describing a system, adding stochasticity and flexibility for these application areas i.e. flows, markets, diffusion and organizations (Bonabeau, 2002). 
Ethical agents was built by Danielson (2003) with a wide variety to satisfy a rich set of functions i.e. learning and reproduction. $\mathrm{ABM}$ was also proposed for modeling ethical behaviors in robots (Dodig-Crnkovic, 2012). Therein, he described Information ethics as "macro-ethics that focuses on systems/ networks of agents and their behavior". More so, the work makes explicit the necessity for all involved with such intelligent technology to assure its ethical conduct. Mclaren (2006) in his work developed agent computational models that produce relevant information and can help humans as they struggle with difficult ethical decisions, as opposed to providing fully supported ethical arguments and conclusions. However, he discussed other forms of computational models for ethical reasoning. Specifically, he analyzed Ethos by Searing (1998), which "encourages rational and consistent ethical problem solving". Also, he reviewed Truth-Teller by McLaren (1995), which "compares pairs of cases presenting ethical dilemmas about whether or not to tell the truth". By the words of Mclaren, one can infer that there is the possibility that, "computational models can be designed to possess the task of autonomously reaching correct conclusions to ethical dilemmas. Having a computer program proposes decisions oversimplifies the obligations of human beings and makes assumptions about the "best" form of ethical reasoning". Considering the arguments that ethical dilemmas sometimes require huge analyses of cause and effect alongside the implicit potential contradictions of a particular behavior, Anderson et al. (2004) built ethical systems that implemented the Act Utilitarianism and Prima Fascia moral reasoning.

Characterizing social media and its attendant issues are a grey area, and not much has been done. However, recently Yulia \& William (2018) modeled human behaviors in responding to emergency situations using social media. The study also involved an integration of a suitable methodology as well as human theories in order to depict unknown emerging patterns. Though their work does not necessarily apply the ABM, it shows the possibility of modeling both social media and human behaviors. In the light of evidences of agent-based modeling for ethical dilemmas presented above, we propose its extension to African work environments where social media and its tools aid interactions and achievement of organizational purposes.

$\mathrm{ABM}$ in the workplace context may strongly relate to the modeling of entrepreneurial behavior proposed by Pyka \& Grebel (2003), where processes may possess some building blocks namely; actors, action, endowments, interaction and evaluation \& decision processes. For actors who may be managers, employees and clients, modelers may conceive the additions of the following; autonomy, social ability, reactivity and proactivity. The actions help in differentiating between one group of actors and another. Put another way, features of actors must differ in order to embody the environment. They described endowments as the "access to material and immaterial resources, their availability together with the individual experiences make up the endowment of the actors". On interaction, the following relationship pairs may be considered; competitive/cooperative, bilateral/multilateral, decentralized/ hierarchical and technological/economic relations. Furthermore, decision making processes of the actors are based on three essential elements which are entrepreneurial, capability-laden and the financial-prone. More so, entrepreneurial decisions are done with social interactions, its attendant social networking dynamics, micro-meso/macro 
feedback, the module/heterogeneous oligopoly and the founding threshold. As DeAngelis \& Diaz (2019) puts it, "the decision-making process of individuals involved in collective movement has been represented by a spectrum of simple to complex rules within ABMs".

In the past, $A B M$ and multi-agent systems (MAS) have been employed to provide support, communication and collaboration in online virtual environments. Logan et al. (2002) built agents that aim to alleviate the innate discomfort of maintaining and keeping tabs with occurrences. These agents keep track of, report, and assist with a variety of responsibilities in a multi-agent environment. The intricacies of affective communications such as facial expressions spurred Neji et al. (2007) to utilize the agent based simulation experiments. Therein, they investigated the way and manner in which social intelligence prompts affective conditions of agents in a virtual collaborative learning environment i.e. how perception of emotion controls virtual encounters for persons and groups. Ren \& Kraut (2014) obtained important ideas from simple theories in social science such as expectancyvalue theory, collective effort model group identity, resource-based and that of UseNet online group etc. Using these they built an agent model for simulating topical breadth, message volume, and moderation. Several benefits were gained from developing ABM/ MAS for online collaborative environments. In Logan et al. (2002) pain was eliminated for the roaming agents, while in the work of Neji et al. (2007) agents represented issues such as distance, entity collaboration and the integration of software elements. Lastly, Ren \& Kraut (2014) allows discovery of the accurate quality/quantity balance as well as the integration of both social and behavioral theories. Even with these benefits, these works may are contextually deficient in the sense that they do not evaluate ethical concerns, does not involve social media and neither was it done for the African environment.

Rudiments of Social Media Exploration using $A B M$

It is noteworthy that providing the requite fundamentals for applying ABM to social media use is necessary in order to guide newbies, enthusiasts and researchers of human computer interaction (HCI), computer supported cooperative work (CSCW) and software engineering to expend research efforts on this subject. Therefore, the requirements of understanding ethical dilemmas in a work environment that employ social media can be summarized using Fig. 1. The potential benefits are better ethical decision making, the creation of better policies, the creation of virtual boundaries as well as continued patronage, if it is a business organization. To arrive at the benefits (right box) of exploring the ethical consequences of social media use in organizations, the analyst/modeler must consider four items below.

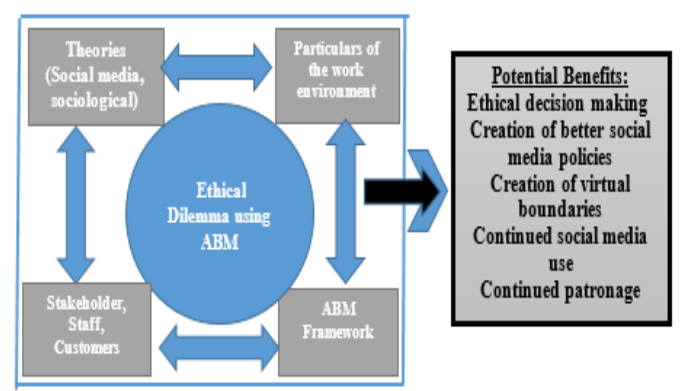

Fig. 1: Exploring Social Media Ethical Dilemmas using $\mathrm{ABM}$.

Social Media Theories: The extant literature on ethical issues in work environment and social media use availed several theories that affect interactions in social networking sites. They include social cognition theory (Jones, 1991) for moral intensity, social learning theory (Bandura, 1986) for leader influence 
on followers, social cognitive domain theory (SCDT) (Turiel, 1983; Turiel, 2002), uses and gratification theory (UGT) (Rubin, 2002) for how individuals use the media. Others include the third person effect and ritualized media use approaches etc. While SCT "considers the unique way in which individuals acquire and maintain behavior, considering the social environment in which individuals perform the behavior" (Jones, 1991), SLT combines the result of learning as a result of psychological factors and learning due to environmental stimuli (Bandura, 1986). On the other hand, according to Davidson (1983), SCDT "was drawn from the philosophical definitions of morality and the psychological research to define morality in terms of obligatory and generalized norms based on concepts of welfare (harm), fairness, rights that regulate social relationships. UGT attempts to the understand the reasons (needs or motivation) behind the use of a particular media as well as its usage consequences (negative and positive). Third-person effect asserts that "people tend to perceive that mass media messages have a greater effect on others than on themselves, based on personal biases" (Davidson, 1983). Aside the social media theories identified above, other sociological such as activity theory, awareness model and behavioral theories have been noted to affect group work (Ren \& Kraut, 2014). The analyst may have to collaborate with researchers from other disciplines to obtain accurate metrics for the formalization of the theories and its usage. Particulars of the work environment: The type of work environment must be considered. A model to be used by people in the academia may differ from the model to be used in organization that sells goods or services to customers.

Stakeholders, the customers and other staff members: $\mathrm{ABM}$ is sometimes referred to as a form of individual based modeling approach. This is because it has the ability to account for "individual level dynamics of a particular phenomenon" (Nwokoye \& Umeh, 2018). Here, the analyst regards the stakeholders, customers and members of staff as agents interacting in an environment. These agents are designed in such a manner that their underlying behavior are taken from the relevant theories and the particulars of the work environment.

ABM/MAS Framework: This refers to the sum total of the agents of the environment, their behavior, the chosen ABM/MAS methodology (discussed in next section) and the choice of programming language (agent toolkit). On the programming tool, some may prefer using a traditional development tools instead of using MadKit and JACK (written in Java). Other development tools including "DEVS (resource centric), DEVS (entity centric), NetLogo, Repast, and SARL" (Leys, 2019).

\section{Methodology for Agent Based Modeling}

Modeling issues in social media would follow the general 4 steps for development of an agentbased model identified by Macal \& North (2006) and Grimm et al. (2010) alongside some insights proposed by Nwokoye, et al. (2018) a modification of the Agent-oriented Software Engineering (Wooldridge \& Ciancarini, 2001). It is well understood that MAS can be interchangeably used in place of ABM (Castiglione, 2009) but other authors posits differences between the two concepts even though they both involve agents interacting in an environment (Rocha et al. 2017). Herein, we lay emphasis on the agent technology which are nonlinear, continuous, possess memory, path-dependence, can learning and adapt. Like in the real world, agents are heterogeneous, stochastic and can allow the representation of a human system such as an organization or workplace. 
Since, operational risk is a reality for any human organization, "a framework that includes the possibility of nonlinear effects because of interactions among subunits and to cascading events" (Bonabeau, 2002) is required. $\mathrm{ABM}$ in its bottom-up simulation approach can work in this manner, operating with the existent lack of historical data. The concept of $\mathrm{ABM}$ is possible with the creation of synthetic environments that mimic the original phenomena. Also, agents are supposed to perceive and cause modifications of this environment (Bandini et al., 2009). In other words, the environment consists of regulations for the control of essential components of the simulated system that are not agents/actors. In fact, they are indications of reference that generate attraction/repulsion forces. The environment plays an important role in the sense that it establishes the general dynamics, thus integrating the effects that have an impact on each agent. In a NetLogo agent language, this synthetic environment is two dimensional, spatial and turtles (agents) can roam around within it. NetLogo also has three dimensional versions representations. In ABM, timescale are discrete time steps which is implemented as pseudorandom numbers in agent oriented languages such as NetLogo (Leys, 2019).

Through understanding ABM experiences and processes we arrived at the following general steps.

Identify the research questions (Grimm, et al., 2010) the model is meant to address. Likely questions include;

a) Can ethical questions concerning social media use be answered using reasoning and analysis with autonomously reactive or proactive agent models?

b) Can modelers reify and thus integrate theories for ABM design and implementation? c) Which dimension of ABM development should African researchers adopt; supporting humans in ethical reasoning or performing autonomous ethical reasoning? Questions may arise from the particulars of the organization and workplace.

Review the relevant literature for theories that highlight the model significance (Grimm et al., 2010). Already, literature avails several theories that affect interactions in social networking sites as well as other sociological or behavioral theories that may affect work environments or group dynamics (Nwokoye et al., 2016).

Identify and design agents, its characteristics (Macal \& North, 2006) and the environment of interaction (Bandini et al., 2009; Leys, 2019). Here, the agents may be the stakeholders involved in decision making at the work place or the employees as well as the customers. Additionally, identified theories can also be characterized in such a manner that they are employed for the success of model design. Tools that may enhance model design may include algorithm (pseudocode or flowchart), graphical user interface controls, layout design and building the data dictionary (Nwokoye \& Umeh, 2018).

Implement the model. The designs are coded using the agent oriented programming approach. Finally, model verification and validation i.e. the model is then tested first for consistency and correctness. Note that the above steps may seem so straightforward, however, the OOD protocol (Grimm et al., 2010) can guide one to implement concepts such as prediction and sensing etc.

Peculiarities of Africa

In ABM studies (Logan et al., 2002; Neji 
et al., 2007), the concept of distance was pinpointed and it presents a strong case for differences noted in several climes. Becoming more curious, we delved into Proxemics (or personal distance) i.e. the distance between individuals as they interact (Hall, 2014). Hall maintained "that differing cultural frameworks for defining and organizing space, which are internalized in all people at an unconscious level, can lead to serious failures of communication and understanding in cross-cultural settings". According to Culture Crossing, what constitutes intimate, personal, social and public distance; which is $0-1.5 \mathrm{ft}, 1.5 \mathrm{ft}-4 \mathrm{ft}, 4 \mathrm{ft}-12 \mathrm{ft}, 12 \mathrm{ft}-25$ $\mathrm{ft}$ respectively for a Nigerian. However, what one may refer to as personal distance can be so different for several cultures. In comparisons between West Africans and Latinos; whereas one loves closer distances in the course of interactions, the other has preference for a larger personal space throughout discussions. There are exceptions when it comes to loved ones. Therefore, in adapting these models or when developing new ones, researchers may have to discover ways to put in attributes of distance for groups, which would allow the perfect representations of more composite social encounters. Additionally, we should also hint at the concept of "African Time" "a metaphorical concept of African time is one in which tardiness, lousiness and a total disregard for schedules and program is made out to characterize all Africans" (Nnajiofor, 2016; Babalola \& Alokan, 2013). Though succinctly argued in his work, Nnajiofor (2016) confirmed that the so-called tardiness and/or lateness is as a result of "the Africans relational attitude, her polychromous nature, even lack of some basic amenities". However, this concept comes into play when designing an ABM for an organizations wherein a manager may need to manage lateness of an employee alongside ethical concerns and other factors of social interaction. On the other hand, the fact that ABM involves the articulation of attendant theories and its organizational effects shows another area of differences in culture. The implication is that the application of theories might prompt underlying variations for several cultures. Ren \& Kraut (2014) confirmed the possibilities of merging various theories from social sciences, behavioral sciences and psychology. More so, theoretical development promised by ABM here prompts African-centric designs and it is important in the progression of HCI inventiveness to African sites. Agent technology would go beyond encouraging theory unification from different disciplines, to characterizing the essence of our daily work in Africa and providing the opportunity for better designs.

\section{Possible challenges and possibilities}

Discovering the right level of abstraction required is the first challenge faced by ABM enthusiasts, although this can be alleviated by developing fine programming skills. Achieving the needed abstraction ensures better implementation and realistic usage. Another challenge is the lack of formalized theories or concepts for ABM applications especially for human behaviors and actions. Bringing it to this discourse, agent-based modeling for ethical reasoning involves "inference rules" which "are available almost exclusively at an abstract level, in the form of principles" (McLaren, 2006). The implication is that modeling ethical behaviors may not be amenable to formal logic used in mathematics or scientific fields. Furthermore, McLaren viewed ethical reasoning as a "weak analytic domain" where rules may involve "open-textured" clauses or phrases which are imprecise, subject to diverse definitions, or possess conflicting meanings in different scenarios.

Another noteworthy challenge in this field of study is the unavailability of materials such 
as research or review papers that explicitly guides potential enthusiasts. An inspection of the above mentioned theories gleaned from social media papers shows that it entails not only social and psychological theories but some mass media theories. Consequently, characterizing these theories would be filled with complexities that may require the collaboration of researchers from not only the computer science fields but persons from other disciplines such as the social and behavioral sciences. Although, ABMs may provide answers to ethical reasoning and dilemma explorations, Sullins (2005) argues that simple agent models may defeat the full evolution of morality unless they are extended to possess cognitive abilities. However, building cognitive ABMs can be very challenging because the researcher must adopt proper (formal) ways of modeling cognitive processes which may still be in its developmental stages. Even with these challenges, we feel the unchartered path of ABM inquiry into ethical dilemmas of social media use (in Africa) is research-worthy. The lack of generally accepted conventions can be eliminated through collaborations with experts in the affected fields of ethical discourses. This would go a long way in developing formalized theories/concepts for ABM representations. It has been discovered that the agent technology can leverage discourses such as CSCW and Postcolonial Computing for the advancement of HCI design and practice in Africa (Nwokoye et al., 2016). The study showed that the applications of agent technology in collaborative systems (such as CSCW) are for the following purposes, which are investigation of team work, collaboration, communication in work places or online virtual environments (CVE). ABM is used to provide answers to hypothetical questions surrounding agents (individual, group, software). The work has also availed the possibilities of reaching ethical conclusions on afro-centric social media usage through agent-based inquiry and reasoning. Conversely, ethical reasoning using ABM can impact pedagogy i.e. teaching students ethical behaviors or teaching employees ethical ways of creating virtual boundaries amidst several overlapping social networks. This implies the building of cognitive tutors or teaching aids but with a bias towards assumptions of Africa. Above, we hinted at theory integration and advancement using agent-based modeling. This is largely possible here. Theories that affect ethical discourses and social media use can be integrated into an autonomous computational agent model with capability of eliciting emergent phenomena.

\section{Conclusion}

The paper attempts to x-ray the not-to-extant literature on representing thus exploring ethical dilemmas of social media using the agent based modeling paradigm. Summarily, this discourse integrates fields such as AI (machine ethics), HCI, psychology, philosophy, simulation and modeling, social and behavioral sciences. The implication is that grasping the overall concept of this discourse may be somewhat daunting for the non-expert. Firstly serving as a guide for enthusiasts and prospective researchers, the study generated the fundamentals of implementing ABM simulations for ethical concerns in workplaces. The overarching and overbearing hypothesis is that stakeholders or policy makers can be modeled as ethical agents interacting in an artificial online collaborative environment. Then, through the evaluation of ABM processes and experiences we derived a 4 step approach for ABM implementation. Some peculiarities noted from investigations were also presented in order to place a hand on dimensions in which enthusiasts may rummage on. Authors identified some elements of differences between Africa and other nations, gleaned insights from social media theories, 
formalize them to suit analyses on how CSCW affect group dynamics by adapting a multiagent simulation framework by Verwaart (2011). Finally, it presents the challenges one may encounter working in these overlapping fields, some opportunities for African work places or organizations in other climes as well as the burning questions that emerge from the discourse.

\section{References}

Anderson, M., Anderson, S. L. \& Armen, C. (2004) Towards Machine Ethics, in Proceedings of AAAI Workshop on Agent Organizations: Theory and Practice, San Jose.

Azar, A. \& Sadeghi, A. (2012) Agent based modeling, a new approach in modeling complex ethical problems. Ethics in Science \& Technology $7,6-16$.

Babalola, S. F. \& Alokan, O. A. (2013) African Concept of Time, a Socio-Cultural Reality in the Process of Change. Journal of Education and Practice, 4, 143 - 147.

Bandini, S., Manzoni, S. \& Vizzari, G. (2009) Agent Based Modeling and Simulation: An Informatics Perspective. Journal of Artificial Societies and Social Simulation 12, 1 - 16.

BAndurA, A. (1986) Social foundations of Thought and Action: A Social Cognitive Theory. Prentice-Hall, Inc.

Bolton, R. N., Parasuraman, A., Hoefnagels, A., Migchels, N. \& KabadaYi, S. (2013) Understanding generation $\mathrm{Y}$ and their use of social media: a review and research agenda. J. of Service Management Marketing Intelligence 24, $328-344$.

Bonabeau, E (2002). Agent-based modeling: Methods and techniques for simulating human systems. PNAS 99, 7280 - 7287.

Castiglione F. (2009) Agent Based Modeling and Simulation, Introduction to Encyclopedia of Complexity and Systems Science. Springer, New York, NY.
DANiELSON, P. (2003) Modeling complex ethical agents. Conference on Computational modeling in the social sciences, Univ. of Washington, Hare.

DAvison, P. W. (1983). Third person effect in communication. Public Opinion Quarterly 47 (1), 1 -15 .

Deangelis, D. L. \& Diaz, S. G. (2019). Decision-Making in Agent-BasedModelin,g: A Current Review and Future Prospectus. Front. Ecol. Evol. 6, $1-15$.

Debatin, B., Lovejoy, J. P., Horn, A. K. \& Hughes, B.N. (2009). Facebook and online privacy: Attitudes, behaviors, and unintended consequences. J. Comp-Med Commun 15, 83 - 108.

Dodig-Crnkovic, G. (2012) Floridi's Information Ethics as Macro-ethics and Info-computational Agent-Based Models BT - Luciano Floridi's Philosophy of Technology: Critical Reflections, in H. Demir (ed.). Springer Netherlands, $3-22$.

Ewokor, C. (2021) Nigeria's Twitter ban: The people risking arrest to tweet. BBC NEWS. https:// www.bbc.com/news/world-africa-57402349.

Grimm, V., Berger, U., Deangelis, D. L., Polhill, G., Giske, J. \& Railsback, S. F. (2010) The odd protocol: A review and first update. Eco. Mod. 221, 2760 - 2768.

Hall, E. T. (1996) Proxemics (Understanding Personal Space). Notes on Intercultural Communication.https://laofutze.wordpress. com/2014/01/03/e-t- hall-proxemics-understanding-personal-space/

JoNES, T. M. (1991) Ethical decision making by individuals in organizations: An issue-contingent model. A. Man. Rev. 16, $366-395$.

Kimball, E. \& Kim, J. R. (2013) Virtual boundaries: ethical considerations for use of social media in social work. Social Work 58, 185 - 188. http://doi.org/10.1093/sw/swt005 
Kunsch, P. L., Kavathatzopoulos, I. \& RauschmaYER, F. (2009) Modelling complex ethical decision problems with operations research. Omega 37, 1100 - 1108. http://doi.org/10.1016/j. omega.2008.11.006

Kunsch, P. L., Theys, M. \& Brans, J. P. (2007) The importance of systems thinking in ethical and sustainable decision-making. Cent. Eur. J. of Operations Research 15, 253 - 269.

Leys, T. (2019) The Current State of Agent-Based Modeling. University of Antwerp, Belgium. Technical Report, 1 - 66 .

Logan, B., Fraser, M., Fielding, D., Benford, S., Greenhalgh, C. \& Herrero, P. (2002) Keeping in touch: Agents reporting from collaborative virtual environments. Technical Report. SS-02-01, AAAI.

Macal, C. M. \& North, M. J. (2006) Tutorial on agent-based modeling and simulation part 2: How to model with agents. In Proceedings of 2006 Winter Simulation Conference, $73-83$.

Mangold, W. G. \& Faulds, D. J. (2009) Social media: the new hybrid element of the promotion mix. Business Horizons 52, 357 - 365.

Mclaren, B. M. (2006) Computational Models of Ethical Reasoning: Challenges, Initial Steps, and Future Directions. IEEE Intelligent Systems 21, 29-37.

Mclaren, B. M. \& Ashley, K. D. (1995) Case-based comparative evaluation in TRUTH-TELLER. The Proceedings from the Seventeenth Annual Conference of the Cognitive Science Society.

Neji, M. \& Ammar, M. B. (2007). Agent-based collaborative affective e-learning framework. The Electronic Journal of e-Learning 5, 123 - 134.

Nnajiofor, O. G. (2016) Justification of the Concept of Time in Africa. Ogirisi: a new journal of African studies 12s, 1 - 30 .

Nwokoye, C. H., Ejiofor, V. E, Orji, R. \& MbeleDoGU, N. (2016) The topicality of agent based modeling/multi agent systems in human computer interaction research: an African perspective. AfriCHI'16 Kenya.

Nwokoye, C. H. \& Umeh, I. (2018) Analytic-agent cyber dynamical systems analysis and design methodology for modeling temporal/spatial factors of malware propagation in wireless sensor networks. Methods X 5, 1373 1398.

Pyka, A. \& Grebel, T. (2003) Agent-based modelling -A methodology for the analysis of qualitative development processes, $1-24$.

Ren, Y. \& Kraut, R. E. (2014) Agent-based modeling to inform online community design: Impact of topical breadth, message volume, and discussion moderation on member commitment and contribution. Human-Computer Interaction 29, $351-389$.

Rocha, J., Boavida-Portugal, I. \& Gomes, E. (2017) Multi-Agent Systems. Intech Open, $1-13$.

Rubin, A.M. (2002) The uses-and-gratifications perspective of media effects.Lawrence Erlbaum Associates Publishers, 2002.

SEARING, D. R. (1998) Harps Ethical Analysis Methodology: Method Description. Taknosys Software Corporation, 1998.

Sullins, J. P. (2005) Ethics and artificial life: From modeling to moral agents. Ethics Inf Technology 7, $139-148$.

Turiel, E. (1983) The Development of Social Knowledge: Morality and Convention. Cambridge University Press, 1983.

Turiel, E. (2002) The Culture of Morality: Social Development, Context, and Conflict. Cambridge University Press.

VerwaART, D. (2011) Agent-based modeling of culture's consequences for trade. Doctoral Thesis. Technical University of Delft.

Voshel, E. H. \& Wesala, A. (2015) Social media \& social work ethics: Determining best practices in an ambiguous reality. Journal of Social Work Values \& Ethics, 12, 67 - 76. 
Wooldridge, M. \& Ciancarini, P. (2001) Agent-oriented software engineering: the state of the art, Agent-oriented Software Engineering, Springer, Berlin Heidelberg, $1-28$.
Yulia, T. \& William, W. (2018) Modeling human behavior on social media in response to significant events. IEEE Transactions on Computational Social Systems, 1 - 14.

Received 02 Jul 20; revised 25 Jun 21. 